\title{
Mounier kuhn syndrome is another rare cause for a long standing dyspnea
}

A 63 y old male with long standing history of dyspnea, and recurrent cough and expectoration was initially thought to be suffering from recurrent lower respiratory tract infection (LRTI) and fibrosing alveolitis. The latest plain chest radiograph showed dilated trachea and bronchi in addition to bilateral diffuse interstitial lung disease. The chest CT scan confirmed the abnormally tracheobronchomegaly which was consistent with the diagnosis of the rare Mounier-Kuhn syndrome of an apparently a sporadic form. Such diagnosis should be considered in the differential diagnosis of settings with radiological findings suggestive of LRTI. The case also emphasizes the importance of the size of air passages on the plain chest radiograph.

\section{KEYWORDS: mounier- kuhn syndrome a tracheobronchomegaly a Recurrent Respiratory Infections (RTIs).}

\section{Case Report}

A 63 y old male with progressive dyspnea for years was suffering from diabetes mellitus, hyperlipidemia and had myocardial infarction followed by mild cerebrovascular accident ten years earlier. He worked as an engineer in oil industry but did not a smoke. Because of the increasing dyspnea, cough and expectoration over the years, he was diagnosed by another facility as having recurrent lower respiratory tract infections (LRTIs) and pulmonary fibrosis (PF) possibly industrial. He was maintained on domiciliary oxygen supplementation in addition to other medication including bronchodilators, anti-lipids, vasodilators, hypoglycemic agents and periodical antibiotics.
Then was put into an early retirement some 3 years ago. The family history was negative of similar illness. He looked significantly, respiratory compromised with dyspnea, cough and expectoration. There were leathery crepitations and scattered rhonchi on chest auscultation. The fingers were clubbed in FIGURE 1. He had no signs suggestive of connective tissue disease. His oxygen saturation ranged between $85 \%$ - 94\%, cardiac ejection fraction $(\mathrm{EF})$ of $33 \%$, and pulmonary artery pressure (PAP) of $42 / 25 \mathrm{~mm} / \mathrm{Hg}$. The lung function test revealed FEV1/FVC (1.20 L/1.22 L) of $98.9 \%$ ), consistent with significant restrictive pattern. The antibody profile for autoimmune disease was negative. A recent DLCO was not carried out.

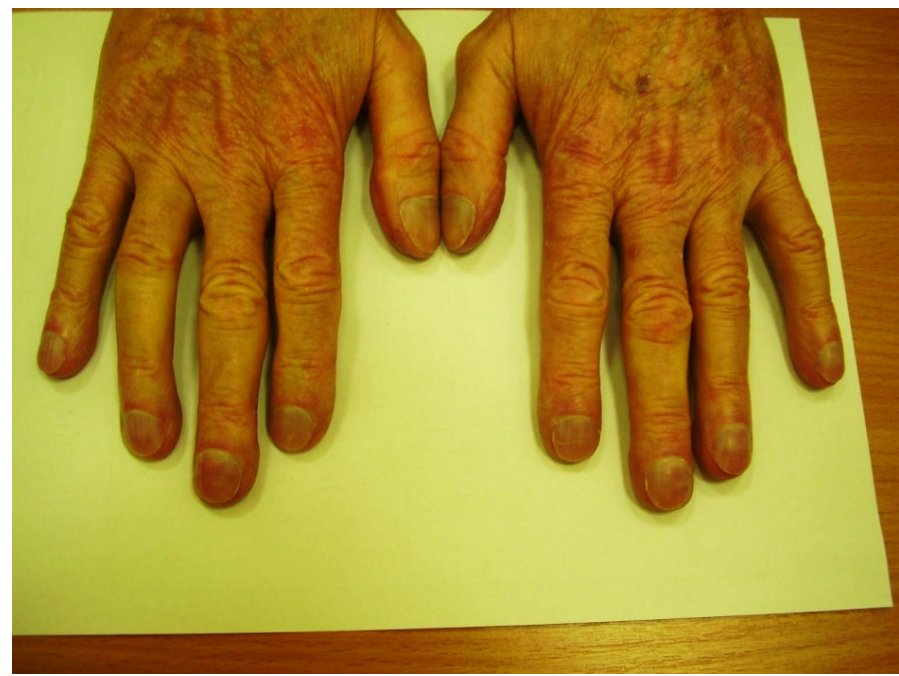

Figure 1. Clubbed and cyanosed fingers. 
The initial plain chest radiograph (FIGURE 2) was reported of diffuse interstitial lung disease pattern more at the bases with extensive honeycombing. However, the trachea and bronchi appeared enlarged. The chest CT scan was diagnostic (FIGURE 3A \& B).

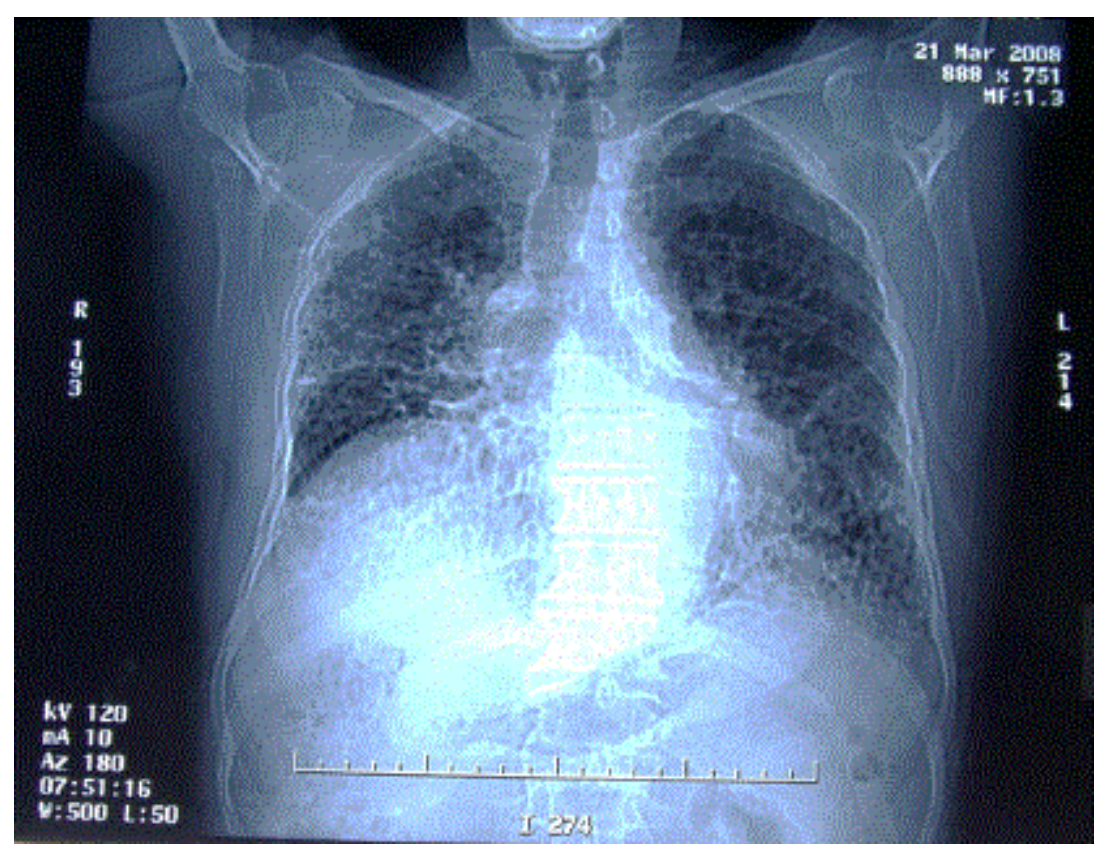

Figure 2. Diffuse interstitial lung disease pattern with honeycombing. Enlarged trachea and bronchi.

It showed bilateral varicoid and cystic bronchiectatic changes with false impression of basal honeycombing at the bases. Enlarged trachea $(3.1 \mathrm{~cm}) \&$ main bronchi $(2.2 \mathrm{~cm})$ demonstrating wall sacculations \& out pouches and Milder interstitial lung markings. Overall it, pointed towards advanced primary airway disease rather than interstitial lung disease (ILD) pattern with extensive bronchiectasis \& tracheobronchomegaly. The features therefore fit with the diagnosis of Mounier Kuhn syndrome.

\section{Discussion}

Mounier Kuhn syndrome or tracheobronchomegaly is a rare clinical and radiologic condition which is characterized by distinct dilatation of the trachea and bronchi and associated with recurrent Lower Respiratory Tract Infections (LTRIs). It is related to atrophy of the muscular and elastic tissues in the trachea and main bronchial wall that leads to pathologic dilation in the tracheobronchial tree and the impairment of mucociliary activity. The presentation varies widely, from a mild disease to a severe respiratory failure. Although its cause is not fully known, yet the familial susceptibility of an autosomal recessive mechanism was suggested. Cases are often sporadic $[1,2]$. Diagnosis of Mounier Kuhn syndrome is often reached by using the computed tomography scan, in which abnormally large air passages are detected. In adults, the diagnostic criteria are diameters of the trachea, $>30 \mathrm{~mm}$; of the right main bronchus, $20 \mathrm{~mm}$; and of the left main bronchus, $18 \mathrm{~mm} \mathrm{[3-6].}$ 

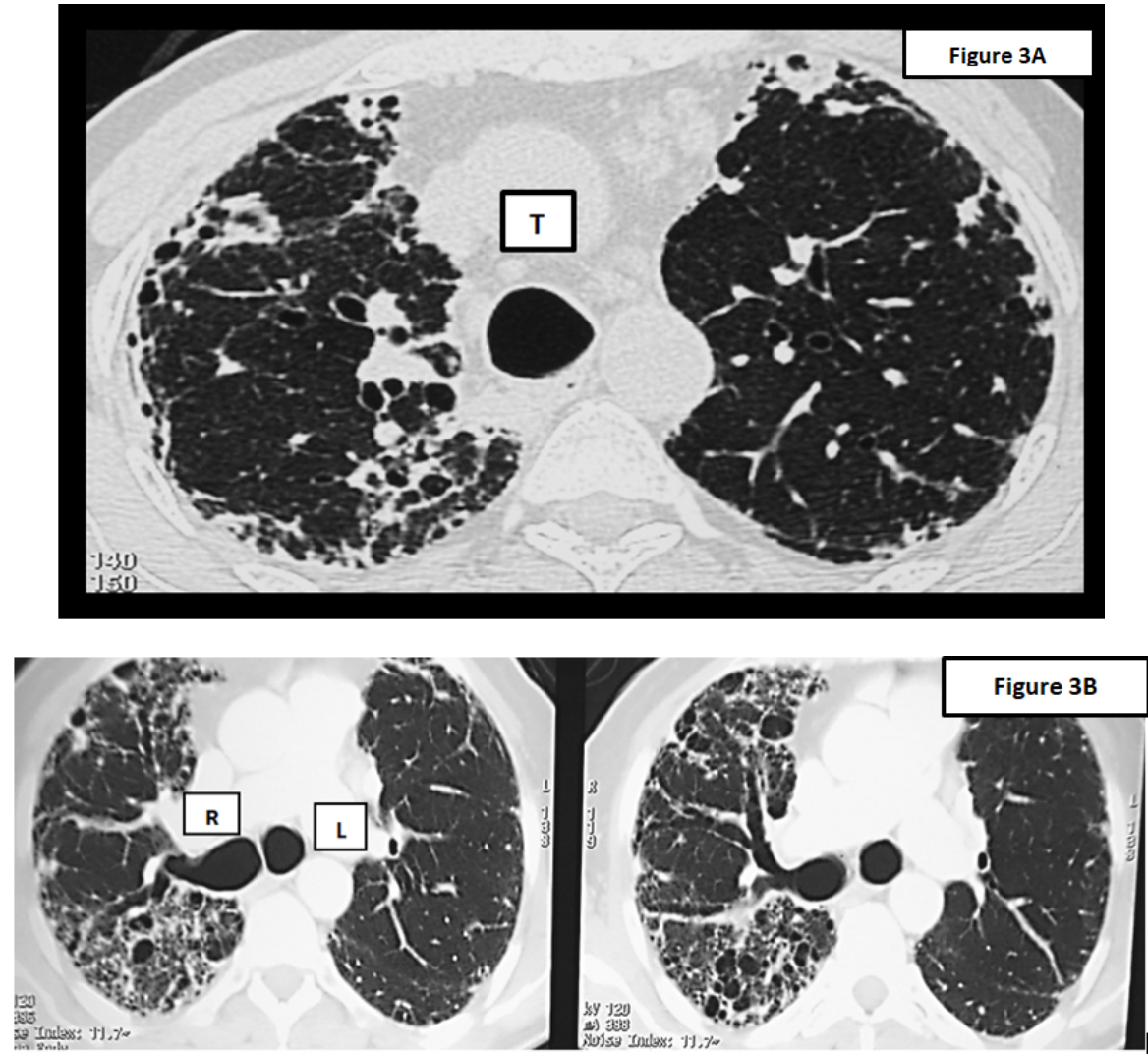

Figure 3. Chest CT scan images.

Bronchography is excellent in demonstrating the extent of disease, but it is becoming obsolete now. A rigid or flexible bronchoscopy can demonstrate the pathologic dilatation of the trachea and main bronchi during inspiration and constriction during expiration and coughing. It is also useful in confirming the diagnosis when CT findings are equivocal; however, it is invasive. Other ancillary investigations to support the diagnosis include pulmonary function testing (PFTs). They demonstrate a reduction in bronchial flow speed, increased tidal volume and dead spaces [6].

A secondary form of tracheobronchomegaly may be seen in ataxia-telangiectasia, ankylosing spondylitis, Ehlers-Danlos syndrome, Marfan syndrome, Kenny-Caffey syndrome, Brachmann-de Lange syndrome, and cutis laxa (elastolysis). These conditions should be considered in the differential diagnosis [3,7].

The treatment is guided by disease severity and the basic aim in all patients is to prevent further damage to the airway tract. This can be achieved by cessation of smoking, minimizing exposure to the industrial and occupational irritants and pollutants. Another important approach in these patients is optimization of concomitant cardiopulmonary conditions such as asthma, chronic obstructive pulmonary disease and chronic bronchitis. Asymptomatic or patients with mild symptoms require no specific treatment. In symptomatic patients, chest physiotherapy allows clearance of secretions. Antibiotics are employed during exacerbations to control infections. Tracheal stenting is helpful in severe cases; however, surgery has virtually little role, considering the diffuse nature of the disease [7] and lung transplantation provided no proved benefit in regard to the risk of morbidity and death [8].

The pulmonary hypertension in the case presented here was secondary to the chronic respiratory disease and the low EF was a sequalae to his coronary artery disease. Nonetheless, the mere initial diagnosis of pulmonary fibrosis was in fact inadequate from the scientific point of view. The clue towards the correct diagnosis of Mounier Kuhn syndrome was the presence of dilated trachea and bronchi seen on the plain X-ray to be followed by the confirmatory CT chest scan. However, despite the rarity of the primary form of tracheobronchomegaly, attention is still to be paid to the size of the air passages since there are conditions other than Mounier 
Kuhn syndrome that might also lead to their enlargement.

Finally, Mounier Kuhn should also be considered in the differential diagnosis in settings with radiological findings suggestive of LRTI.

Overall, our patient had a rather complicated disease in addition to the presence of other comorbid conditions including diabetes, cerebrovascular and coronary artery disease. That all led to his expiry due to cardiopulmonary failure months after diagnosis of Mounier Khun syndrome had been made.

\section{References}

1. Celik B, Bilgin S, Yuksel C. MounierKuhn syndrome, a rare cause of bronchial dilation. Tex. Heart. Inst. J. 38, 194-196, (2011).

2. Damgaci L, Durmus S, Pasaoglu E. Mounier-Kuhn syndrome (tracheobronchomegaly). Tanisal. Ve. Girisimsel. Radyoloji. 8, 165-166, (2002).

3. Menon B, Aggarwal B, Iqbal A. Mounier-Kuhn syndrome: report of 8 cases of tracheobronchomegaly with associated complications. South. Med. J. 101, 83-87, (2008).

4. Ghanei M, Peyman M, Aslani J et al. Mounier-Kuhn syndrome: a rare cause of severe bronchial dilatation with normal pulmonary function test: a case report. Respir. Med. 101, 1836-1839, (2007).

5. Dunne MG, Reiner B. CT features of tracheobronchomegaly. J. Comput. Assist. Tomogr. 12, 388-391, (1988).

6. Gupt KP, Gorsi U, Bhalla A et al. Mounier Khun syndrome masquerading pulmonary thromboembolism in an elderly male. Lung. India. 31: 76-78, (2014)

7. Ernst A, Majid A, Feller-Kopman D et al. Airway stabilization with silicone stents for treating adult tracheobronchomalacia: A prospective observational study. Chest. 132: 609-616, (2007).

8. Noori F, Abduljawad S, Suffin DM et al. Mounier-Kuhn syndrome: a case report. Lung. 188, 353-354, (2010). 